\title{
Scan-Time Reduction Using Noise-Matched Images in 2- and 3-Dimensional Bismuth Germanate PET/CT: Clinical Study in Head and Neck Cancer
}

\author{
Bal Sanghera ${ }^{1}$, Gerry Lowe ${ }^{2}$, David Wellstead ${ }^{3}$, John Lowe $^{1}$, Jane Chambers ${ }^{1}$, and WaiLup Wong ${ }^{1}$ \\ ${ }^{1}$ Paul Strickland Scanner Centre, Mount Vernon Hospital, Northwood, Middlesex, United Kingdom; ${ }^{2}$ Mount Vernon Hospital, \\ Northwood, Middlesex, United Kingdom; and ${ }^{3}$ Health Research and Development Support Unit, University of Hertfordshire, \\ Hertfordshire, United Kingdom
}

\begin{abstract}
We quantitatively and qualitatively investigated 2-dimensional (2D) and 3-dimensional (3D) imaging with scan-time reduction in 14 patients with 17 lesions, who had known or suspected head and neck cancer, using a bismuth germanate (BGO) crystal based PET/CT scanner with noise-matched images. Methods: A 2D and 3D acquisition protocol using scan-time reduction on an axial single field of view resulted in a 2D 4-, 3D 4-, 3D 3-, 2D 3-, 2D 2-, and $3 D$ 2-min scan sequence to minimize redistribution and decay bias. Tumor maximum standardized uptake values (SUVmax) and tumor mean standardized uptake values $\left(\mathrm{SUV}_{\text {mean }}\right)$ were recorded, and two observers in consensus investigated lesion conspicuity between 2D and 3D paired 4-, 3-, and 2-min noisematched images. Results: We found some minor advantages quantitatively in favor of 2D scanning, with higher mean SUVmax, and qualitatively in favor of 3D scanning, with lesion conspicuity preference. In our cohort, no great advantage or disadvantage to using either acquisition mode was observed, and all lesions were seen irrespective of acquisition mode and scan time. Conclusion: In head and neck cancer patients, we can recommend a scan-time reduction from 4 to $3 \mathrm{~min} /$ bed position in 2D acquisitions with a BGO-based PET/CT scanner, using our imaging protocol and reconstruction defaults.
\end{abstract}

Key Words: PET/CT; 2D/3D; comparison; scan time; reduction

J Nucl Med Technol 2009; 37:74-82

DOI: $10.2967 /$ jnmt.108.055855

\section{B} ismuth germanate (BGO) crystal-based PET and more recent combined PET/CT scanners are in widespread clinical use, predominantly to image ${ }^{18} \mathrm{~F}-\mathrm{FDG}$ in cancer studies, but there is still controversy over potential advantages or disadvantages of 2-dimensional (2D) versus 3-dimensional (3D)

\footnotetext{
Received Jul. 10, 2008; revision accepted Feb. 20, 2009.

For correspondence or reprints contact: Bal Sanghera, Paul Strickland Scanner Centre, Mount Vernon Hospital, Northwood, HA6 2RN United Kingdom.

E-mail: bal.sanghera@nhs.net

COPYRIGHT @ 2009 by the Society of Nuclear Medicine, Inc.
}

acquisition and scan-time reduction clinically. In 2D scanning, interplane septa reduce image-degrading random and scattered counts at the expense of overall system sensitivity, with the possibility of long scan times to improve count statistics. In 3D scanning, septa are retracted (typically allowing a large increase in such counts), thereby requiring imaging systems with characteristics of low dead time, fast decay crystal or readout electronics, good energy resolution, and scatter correction to optimize the signal-to-noise ratio in images.

Such factors raise questions about the clinical advantages and disadvantages of $2 \mathrm{D}$ acquisition, compared with $3 \mathrm{D}$ acquisition (1), regarding image quality and potential scantime and dose reduction. Additional parameters that may influence this comparison are patient demographics, fasting time before scanning, choice and amount of tracer administered, time to start scanning after injection, data representation and reconstruction algorithms used (2), scanner used (3), and type or site of cancer.

Phantom studies have been used to investigate the lesionto-background ratios for $2 \mathrm{D}$ and $3 \mathrm{D}$ acquisition modes in whole-body (WB) scanning. In 1 phantom study, injected activity was optimized using $2 \mathrm{D}$ and $3 \mathrm{D}$ noise equivalent counting (NEC) rates, but no advantage was seen (4). Similarly, no improvement was found between scanning modes in a small tumor or lymph node phantom study (5). However, 3D imaging was favored when the injected activity was optimized for this mode, but 2D imaging was found to be superior for detecting lesions at higher doses (6).

This uncertainty led some (7) to investigate the optimization of the clinical performance of a BGO-based scanner by interleaving $2 \mathrm{D}$ (scan duration, $5 \mathrm{~min} /$ bed position) acquisitions with 3D (scan duration, 3, 4, and $5 \mathrm{~min} /$ bed position) acquisitions in WB clinical acquisitions, with phantom studies used to optimize 2D and 3D imaging parameters.

Matching noise in paired 2D and 3D scans, by the application of postreconstruction filtering, can reduce bias in uptake measurements arising through differences in 2D and 
$3 D$ reconstruction techniques. A 2D versus 3D simulated WB clinical study was performed with dynamic acquisitions on a lutetium oxyorthosilicate-based scanner using a single field of view (SFOV) (8) with noise-matched images. Transmission scanning was performed using ${ }^{68} \mathrm{Ge}$ rod sources, as in the studies by Visvikis et al. (7) and Lodge et al. (8); each of these publications concluded that 3 dimensions offered clinical image quality comparable to, if not better than, 2 dimensions.

Emphasis has also been placed on 2D versus 3D comparison, with imaging optimization on specific tumor sites rather than general WB scanning. In 1 such brain study, Dhawan et al. (9) used an interleaved 2D and 3D protocol for acquiring sequential scans and found that $3 \mathrm{D}{ }^{18} \mathrm{~F}$-FDG studies were comparable to half the injected activity or scan duration of similar 2D scans. However, different conclusions were made in high-counting-rate cardiac studies regarding the advantage, if any (10), of 2D (11) versus 3D acquisition (12). Following recent reports on WB scanning (13), advances in reconstruction algorithms on the latest BGO-based scanners have led to advantages in $3 \mathrm{D}$ over $2 \mathrm{D}$ acquisition for the imaging of specific lesions. However, some ambiguity exists in the literature regarding the potential clinical benefits of site-dependent $2 \mathrm{D}$ versus $3 \mathrm{D}$ imaging. This effect may be more pronounced in older BGO systems with moderate crystal energy resolution, relatively long decay times, and less sophisticated image reconstruction algorithm implementation. Our institution has clinical experience in ${ }^{18} \mathrm{~F}-\mathrm{FDG}$ head and neck cancer studies and research (14) and associated therapies (15). Accordingly, we investigated the advantages and disadvantages of $2 \mathrm{D}$ versus $3 \mathrm{D}$ acquisitions and scan-time reduction on a PET/CT BGO crystal scanner (Discovery ST; GE Healthcare) using paired 4-, 3-, and 2-min scan times on an SFOV.

Our aim was to investigate lesion maximum standardized uptake values (SUVmax) and lesion mean standardized uptake values (SUVmean) for each respective 2D and 3D scan pair and for each scan time under conditions of matched noise, thus minimizing $2 \mathrm{D}$ and $3 \mathrm{D}$ reconstruction bias effects. Two experienced observers then investigated the clinical significance of these quantitative results by performing a qualitative visual comparison of lesion conspicuity between paired scans.

\section{MATERIALS AND METHODS}

Informed written consent was obtained from 14 patients with known or suspected head and neck cancer (total of 17 lesions) who were recruited into this prospective, single-institution, nonrandomized study approved by the local Mount Vernon Ethics Committee.

Our cohort consisted of 12 men and 2 women (average age \pm SD, $51 \pm 13 \mathrm{y}$; average weight $\pm \mathrm{SD}, 75 \pm 12 \mathrm{~kg}$ ). Patients fasted for at least $6 \mathrm{~h}$ before an intravenous administration of ${ }^{18} \mathrm{~F}-\mathrm{FDG}$ (4.5 MBq/kg of body weight). WB scans began on average $65 \pm$ 6 min after the injection of the tracer, and the average ${ }^{18} \mathrm{~F}-\mathrm{FDG}$ uptake time to the first SFOV scan (i.e., in 2 dimensions for 4 min) was $92 \pm 6 \mathrm{~min}$.
The entire scanning protocol comprised WB PET/CT from the base of the brain to the groin. All patients were scanned with their arms down by their sides to reduce noise in head and neck images. The following were typical WB, spiral, 4-slice CT parameters: 0.8-s tube rotation, $140 \mathrm{kVp}, 80 \mathrm{~mA}$, and 1.5:1 pitch. Routine 2DWB PET started approximately $60 \mathrm{~min}$ after injection, with a duration of $4 \mathrm{~min} / \mathrm{bed}$ position and an overlap of 3 slices.

Reconstructed PET/CT WB images were used to locate head and neck lesions for calculating bed travel distances, to position the highest lesion uptake region in the PET SFOV center. In this way, bias from nonuniform sensitivity across the 3D FOV for interleaved scans that followed was minimized. After a regular WB scan, the patient continued to lie still as the bed position was translated the required distance; paired, interleaved 2D and 3D PET images were acquired for each scan time (Fig. 1).

\section{Image Reconstruction}

The National Electrical Manufacturers Association/International Electrotechnical Commission (NEMA/IEC) 2000 PET phantom, consisting of 6 spheres (10-, 13-, 17-, 22-, 28-, and $37-\mathrm{mm}$ diameters) situated in a background phantom, was used to deduce optimized reconstruction parameters for SFOV 2D and 3D scanning. A clinically realistic hot sphere-to-background activity ratio of approximately 4:1 was used, and reconstruction parameters were considered optimized when the hot sphere SUVmax was close to or had reached a plateau signifying convergence in the reconstruction.

All 2D and 3D SFOV PET transaxial emission images were reconstructed to a $128 \times 128 \times 47$ matrix, with pixel dimensions of $4.68 \times 4.68 \times 3.27 \mathrm{~mm} .2 \mathrm{D}$ reconstructions were performed using an ordered-subset expectation maximization (OSEM) iterative algorithm (16) with scatter correction (17). 3D reconstructions

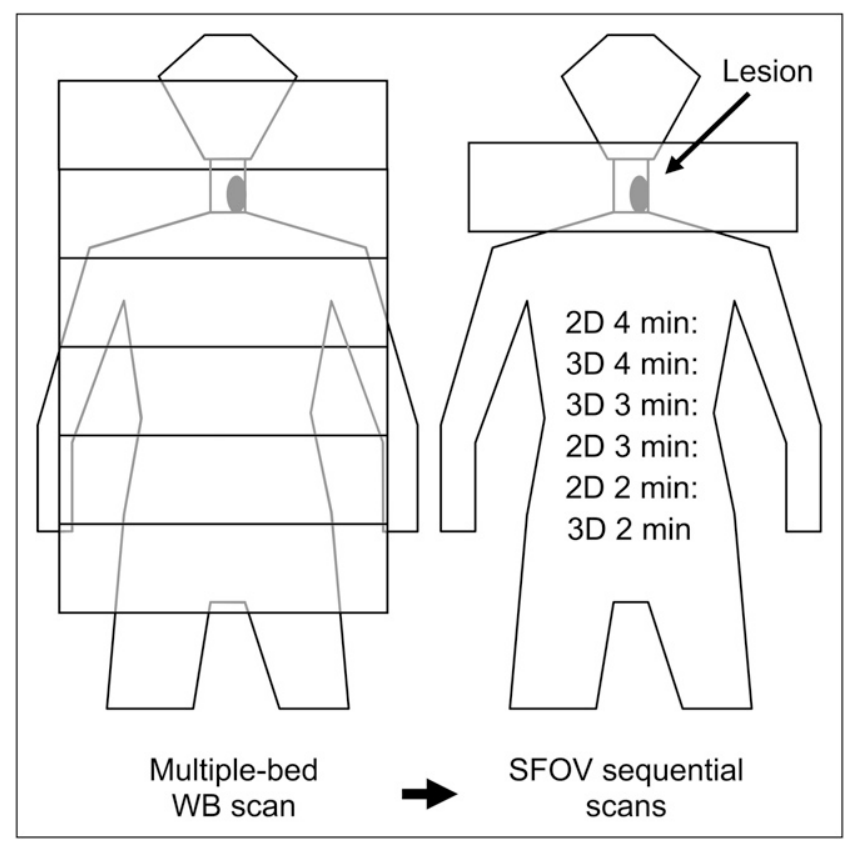

FIGURE 1. Procedure used to investigate acquisition mode and scan time in head and neck cancer patients. Protocol allowed standard WB scanning to be performed, followed by optimized SFOV scans, without extra radiation burden to subject. 
used Fourier rebinning, followed by weighted least-squares iterative reconstruction OSEM (18) and model-based scatter correction (19). Additional 2D and 3D corrections were performed for randoms (singles), decay, dead time, geometry, and attenuation.

\section{Image Analysis}

For each patient, a predominantly circular region of interest (ROI) was drawn by hand using the scanner manufacturer's imaging workstation-integrated software to encompass the tumor, thus allowing SUVmax and SUVmean to be recorded on each patient's 2D 4-min SFOV axial scan. An ROI was carefully positioned close to the lesion in the same image slice, to acquire a uniform mean background uptake. The percentage coefficient of variation was defined as 100 multiplied by (SD/mean) of the average background ROI counts; this parameter was used as a quantitative measure of image noise. To minimize variability, the same lesion and background ROIs were then applied in the same locations to each of the other scans composed of different acquisition modes and times.

Our study incorporated noise-matched scans to improve efficacy. For every patient, each 2D or 3D SFOV acquisition was reconstructed using a gaussian postreconstruction filter of 1-, 2-, 3-, 4.5-, 5.14-, 6-, 7-, 8-, 9-, and 10-mm full width at half maximum, resulting in 10 different reconstructed scans for each 2D or 3D SFOV acquisition. For each reconstructed scan, a noise value, established close to the lesion SUVmax, was recorded using the same background ROI, resulting in a total of 10 noise values for each $2 \mathrm{D}$ or $3 \mathrm{D}$ SFOV acquisition. We compared each of these 10 noise values from the $2 \mathrm{D}$ acquisition with each of the 10 noise values from the paired 3D acquisition for each SFOV (e.g., 2D $4 \mathrm{~min}$ and 3D $4 \mathrm{~min}$ ) to establish the closest noise match. The $2 \mathrm{D}$ and $3 \mathrm{D}$ respective lesion SUVmax and SUVmean were then recorded. This process was repeated, ensuring that noise match was specific to each patient's paired 2D and 3D acquisition and specific to each 4-, 3-, and 2-min scan.

For qualitative analysis, each noise-matched pair of 2D and 3D scans acquired for 4, 3, and 2 min was compared for lesion conspicuity by 2 experienced radiologists in joint consensus and rated accordingly with a score of $-2,-1,0,+1$, or +2 , in preference for 1 of the 2 scans. A score of 0 signified that no preference was expressed for either scan by the observers, a score of -1 or +1 implied that one scan appeared superior to the other, and a score of -2 or +2 suggested that one scan appeared far superior to the other.

\section{Statistical Analysis}

SUVmax and SUVmean data acquired in 2 and 3 dimensions for different scan times were positively skewed, requiring $\log _{10}$ transformation to approximate gaussian behavior for statistical analysis. The Shapiro-Wilk test is preferred for investigating normality in small sample size distributions and was used in this study.

Linear regression and the Pearson correlation coefficient were used to investigate the possibility of bias in results introduced through correlations between SUVmax with weight, height, body mass index (BMI), and blood glucose.

Differences between images for recorded SUVmax at each scan time were analyzed using the paired $t$ test; a $P$ value of less than 0.05 was considered statistically significant, and a $P$ value of less than 0.0001 was considered highly statistically significant. The same was also true for SUVmean and noise measurements, respectively, in paired scans.

For qualitative analysis, the scoring system resulted in a preference scale that was parametric, for which a mean and $95 \%$ confi- dence interval $(\mathrm{CI})$ were constructed. A consistent preference for 1 scan in the direction indicated by the coding at a $5 \%$ level was suggested when the $95 \% \mathrm{CI}$ did not cross 0 and was consistent with a 1 -sample $t$ test.

\section{RESULTS}

\section{D and 3D Reconstruction Parameters}

Figure 2 shows SUVmax normalized to the maximum for each acquisition mode and scan duration using the 28-mmdiameter sphere in the NEMA/IEC 2000 PET phantom and illustrates increased uptake with increasing OSEM number of iterations multiplied by subsets in 2D and 3D image reconstruction. The same trend of hot sphere uptake reaching a plateau with increased number of reconstruction iterations multiplied by subsets was observed with the other 5 spheres in the phantom.

We selected 21 iterations and 30 subsets (iterations $\times$ subsets, 630) for optimized $2 \mathrm{D}$ reconstruction and 20 iterations and 32 subsets (iterations $\times$ subsets, 640) for optimized 3D reconstruction, based on SUVmax from all spheres reaching or being close to the plateau to ensure convergence.

\section{Noise Match in Paired Scans}

Figure 3 shows the distribution of noise-matched data between 4-, 3-, and 2-min paired 2D and 3D images for each patient. Results from this noise-matched plot are presented in Table 1, which shows the mean noise value, SD, difference in means, and associated statistical significance for paired scans of different scan durations. The nonsignificant differences

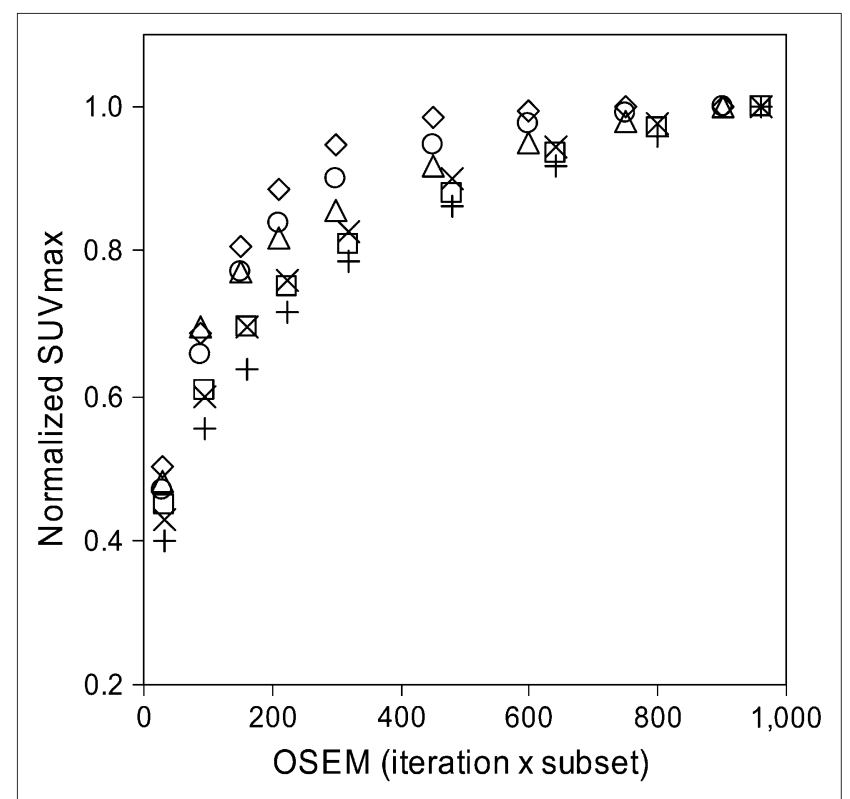

FIGURE 2. Normalized SUVmax versus OSEM (iteration $\times$ subset) acquired with hot sphere-to-background ratio of $4: 1$, showing convergence of $2 \mathrm{D}$ and $3 \mathrm{D}$ acquisition modes. $\diamond=2 \mathrm{D}$ $4 \mathrm{~min} ; O=2 \mathrm{D} 3 \mathrm{~min} ; \triangle=2 \mathrm{D} 2 \mathrm{~min} ; \square=3 \mathrm{D} 4 \mathrm{~min} ; \times=3 \mathrm{D}$ $3 \mathrm{~min} ;++=3 \mathrm{D} 2 \mathrm{~min}$. 


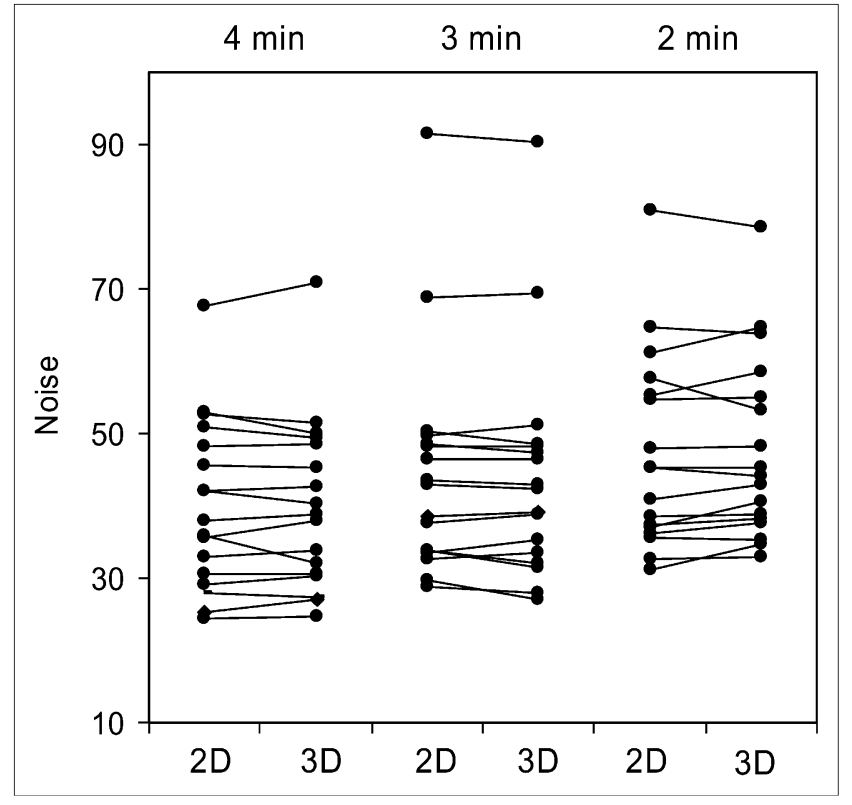

FIGURE 3. Plot of noise matching in paired 2D and 3D acquisitions with different scan times. Difference in noise means between 4-min 2D acquisition and 4-min 3D acquisition was 0.02 and not significant $(P=0.97)$. This was the case for 3 -min $(P=$ $0.17)$ and, likewise, 2 -min acquisitions $(P=0.25)$, showing close match in noise between paired scans.

observed for 4-, 3-, and 2-min scans are desirable, suggesting a close match in noise characteristics between 2D and 3D paired scans.

\section{SUVmean in Paired Scans}

Figure 4 illustrates the distribution in SUVmean data between paired 2D and 3D images for each patient with 4-, 3 -, and 2-min scans. Results from this SUVmean plot are presented in Table 2, which shows the mean SUVmean, SD, difference in means, and associated statistical significance

TABLE 1.

Results of Matching Noise in Paired 2D and 3D Acquisitions

\begin{tabular}{ccccc}
\hline Paired scan & Mean noise (\%) & SD & Difference & $P$ \\
\hline 4 min & & & 0.02 & 0.97 \\
2D & 38.41 & 9.65 & & \\
3D & 38.39 & 8.67 & & \\
3 min & & & 0.51 & 0.17 \\
3D & 39.81 & 8.18 & & \\
2D & 40.32 & 7.67 & & \\
2 min & & & 0.67 & 0.25 \\
2D & 44.65 & 10.21 & & \\
3D & 45.32 & 9.33 & &
\end{tabular}

No significant difference was seen in mean noise between $2 \mathrm{D}$ and 3D paired scans of 4,3 , and 2 min, showing that good noisematched imaging was achieved.

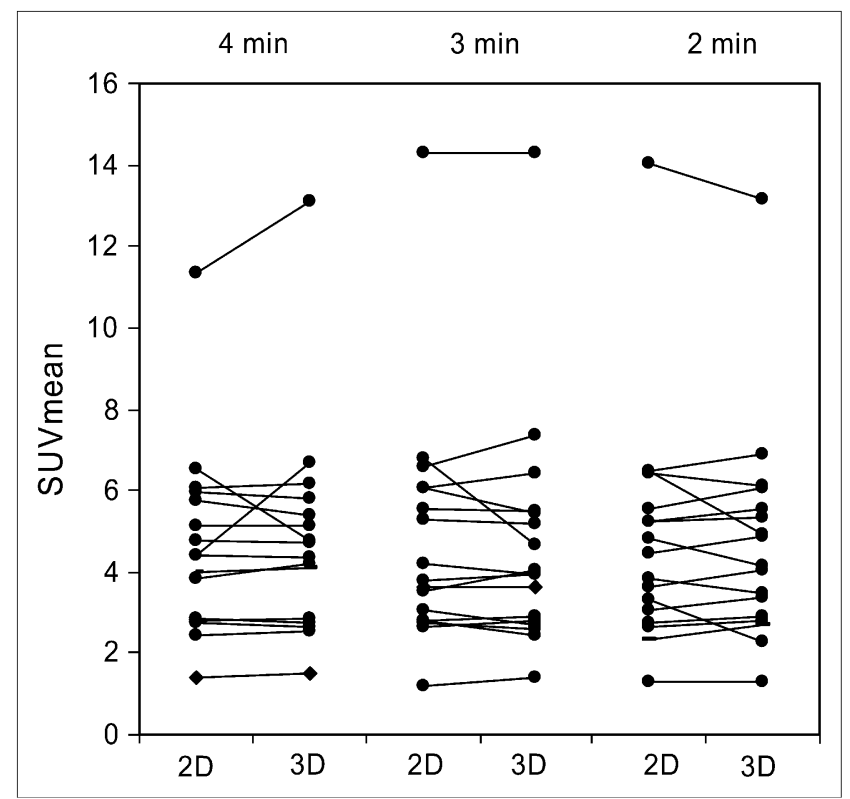

FIGURE 4. Plot of lesion SUVmean recorded for 2D and 3D acquisitions with different scan times. Difference in mean SUVmean between 4-min 2D acquisition and 4-min 3D acquisition was 0.14 and not significant $(P=0.54)$. This was the case for 3-min $(P=0.55)$ and, likewise, 2-min acquisitions ( $P=0.45)$, showing no great superiority of either mode with any scan times.

for paired scans of different scan durations. Nonsignificant differences are reported for each scan pair.

\section{SUVmax in Paired Scans}

Figure 5 depicts the distribution in SUVmax data between paired 2D and 3D images for each patient with 4-, 3-, and 2-min scans. Results from this SUVmax plot are presented in Table 3, which shows the mean SUVmax, SD, difference in means, and associated statistical significance for paired scans of different scan durations. A significant difference for only

TABLE 2

Results of Mean SUVmean in Paired 2D and 3D Acquisitions

\begin{tabular}{ccccc}
\hline Paired scan & Mean SUVmean & SD & Difference & $P$ \\
\hline 4 min & & & 0.14 & 0.54 \\
2D & 4.55 & 2.28 & & \\
3D & 4.69 & 2.6 & & \\
3 min & & & 0.11 & 0.55 \\
3D & 4.66 & 2.93 & & \\
2D & 4.77 & 2.94 & & \\
2 min & & & 0.09 & 0.45 \\
2D & 4.79 & 2.84 & & \\
3D & 4.7 & 2.67 & &
\end{tabular}

No significant difference was seen in mean SUVmean between $2 \mathrm{D}$ and $3 \mathrm{D}$ paired scans of 4,3 , and $2 \mathrm{~min}$, suggesting no great superiority of either paired acquisition mode with scan duration. 


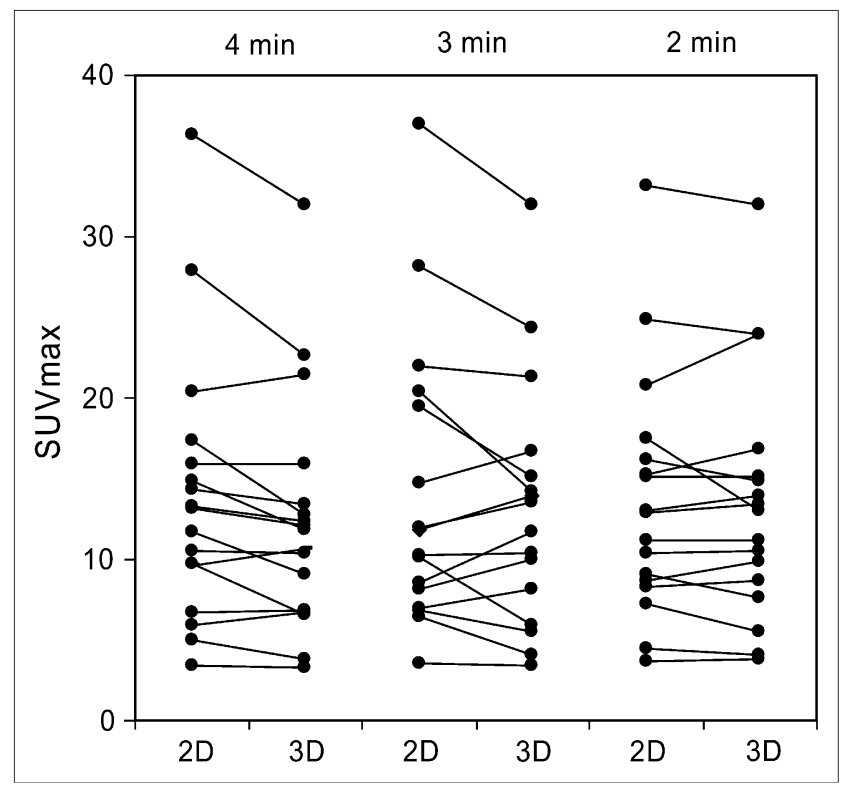

FIGURE 5. Plot of lesion SUVmax recorded for 2D and 3D acquisitions with different scan times. Difference in uptake means between 4-min 2D acquisition and 4-min 3D acquisition was 1.43 and significant $(P=0.01)$. However, nonsignificant results were obtained for 3-min $(P=0.17)$ and, likewise, 2-min acquisitions $(P=0.57)$.

the paired 2D 4-min and 3D 4-min scans (95\% CI, 0.03-0.18; paired $t$ test, $t=2.90, P=0.01$ ) was observed.

\section{Qualitative Observer Scores}

Figure 6 depicts SFOV noise-matched axial slices that contain the SUVmax from 1 patient, acquired with 4-, 3-, and 2-min scans.

The observer scores for preference in paired scans are shown in Table 4.

Qualitative results for observer-scored paired scans using the 1-sample $t$ test are also shown in Table 4. The mean, SE, 95\% CI, and statistical significance of the score between paired scans are depicted. Consensus agreement between

TABLE 3.

Results of Mean SUVmax in Paired 2D and 3D Acquisitions

\begin{tabular}{ccccc}
\hline Paired scan & Mean SUVmax & SD & Difference & $P$ \\
\hline 4 min & & & 1.43 & 0.01 \\
2D & 13.89 & 8.35 & & \\
3D & 12.46 & 7.31 & & \\
3 min & & & 1.37 & 0.17 \\
3D & 13.10 & 7.49 & & \\
2D & 14.47 & 8.86 & & \\
2 min & & & 0.2 & 0.57 \\
2D & 13.64 & 7.5 & & \\
3D & 13.44 & 7.52 & &
\end{tabular}

Significant difference was seen only in mean SUVmax for 2D and $3 \mathrm{D}$ paired scans of $4 \mathrm{~min}$, suggesting some superiority for $2 \mathrm{D}$ scans.
A

C
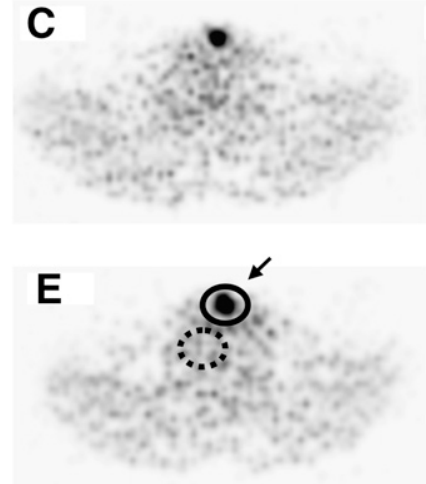

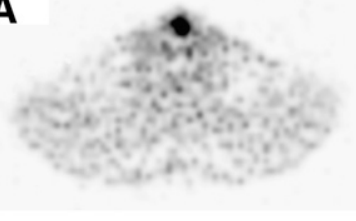

B

B

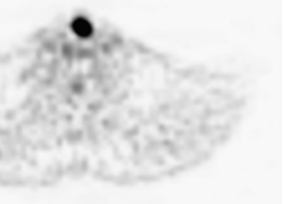

D

$\mathbf{F}$
FIGURE 6. Example of noise-matched images from patient. Images depicted are from 2D 4-min (A), 3D 4-min (B), 2D 3-min (C), 3D 3-min (D), 2D 2-min (E), and 3D 2-min (F) scans. Arrow indicates lesion, and ROls were used to define lesion SUVmax or SUVmean (solid circle in E) and uniform background area (dotted circle in E) for semiquantitative analysis.

experienced radiologists resulted in a significant preference for the conspicuity of lesions in paired 3D 3-min scans, compared with 2D 3-min scans, and in paired 3D 4-min scans, compared with 2D 4-min scans, only.

\section{SUVmax Correlations}

No statistically significant correlation between age at the time of the scan, weight, height, BMI, and blood glucose with SUVmax was observed for this cohort of patients. Table 5 shows the respective $P$ values calculated for 2D and 3D 4-, 3-, and 2-min scans for these parameters, calculated using linear regression and the Pearson correlation coefficient.

\section{DISCUSSION}

In this study, we focused on genuine, consecutive patients with suspected head and neck cancer who had routine clinical WB scans in our center using standard protocols. No attempt was made to confirm diagnosis with histology. Similar to another study (13), our protocol allowed WB scans to be obtained under standard imaging conditions for radiologists; 2D and 3D research SFOV images with reduced scan times were then acquired if the patient agreed to continue. Our protocol provided not only valuable information about the advantages and disadvantages of 2 dimensions versus 3 dimensions with scan-time reduction in head and neck cancer but also a potential clinical benefit of optimized scanning on those occasions in which more head and neck patients require scanning in a restricted time period. This benefit is particularly true in situations in which delays occurred (e.g., because of machine failure or reduced ${ }^{18} \mathrm{~F}-\mathrm{FDG}$ supply or the like). 
TABLE 4.

Observer Scores of Preference in Paired Scans with Statistical Significance

\begin{tabular}{|c|c|c|c|c|c|c|c|c|c|}
\hline Patient no. & $\begin{array}{c}\text { 2D } 2 \\
\text { min vs. } \\
\text { 2D } 4 \mathrm{~min}\end{array}$ & $\begin{array}{c}\text { 2D } 2 \\
\text { min vs. } \\
2 \mathrm{D} 3 \mathrm{~min}\end{array}$ & $\begin{array}{c}\text { 2D } 3 \\
\text { min vs. } \\
2 \mathrm{D} 4 \mathrm{~min}\end{array}$ & $\begin{array}{c}3 \mathrm{D} 2 \\
\text { min vs. } \\
\text { 3D } 4 \mathrm{~min}\end{array}$ & $\begin{array}{c}3 \mathrm{D} 2 \\
\text { min vs. } \\
\text { 3D } 3 \mathrm{~min}\end{array}$ & $\begin{array}{c}\text { 3D } 3 \\
\text { min vs. } \\
\text { 3D } 4 \text { min }\end{array}$ & $\begin{array}{c}2 \mathrm{D} 2 \\
\min \mathrm{vs} . \\
3 \mathrm{D} 2 \mathrm{~min}\end{array}$ & $\begin{array}{c}2 \mathrm{D} 3 \\
\text { min vs. } \\
\text { 3D } 3 \mathrm{~min}\end{array}$ & $\begin{array}{c}\text { 2D } 4 \\
\text { min vs. } \\
\text { 3D } 4 \mathrm{~min}\end{array}$ \\
\hline 1 & -1 & 0 & -1 & +2 & +1 & +1 & 0 & +1 & +2 \\
\hline 2 & -1 & 0 & +1 & -1 & 0 & -1 & -1 & +2 & -1 \\
\hline 3 & 0 & 0 & 0 & -1 & 0 & +1 & 0 & +2 & +1 \\
\hline 4 & +1 & +1 & -1 & +1 & +1 & 0 & +2 & +2 & +1 \\
\hline 5 & -1 & 0 & 0 & +2 & -1 & 0 & +1 & +2 & +2 \\
\hline 6 & -1 & -1 & 0 & +1 & -1 & -1 & +1 & +1 & +1 \\
\hline 7 & +2 & +1 & 0 & -2 & +1 & -1 & -1 & +2 & +1 \\
\hline 8 & +1 & -1 & -1 & -1 & -1 & +1 & +1 & +1 & +1 \\
\hline 9 & -2 & -1 & 0 & 0 & 0 & 0 & 0 & +2 & +2 \\
\hline 10 & +2 & +2 & +1 & +2 & +1 & +1 & -2 & +1 & +2 \\
\hline 11 & 0 & +1 & -1 & -1 & 0 & 0 & +1 & +1 & +2 \\
\hline 12 & 0 & -2 & +1 & 0 & 0 & +1 & -1 & +1 & +1 \\
\hline 13 & +2 & +1 & +2 & +1 & +1 & +1 & 0 & +1 & +1 \\
\hline 14 & 0 & 0 & 0 & 0 & 0 & 0 & 0 & 0 & 0 \\
\hline Mean & 0.14 & 0.07 & 0.07 & 0.21 & 0.14 & 0.21 & 0.07 & 1.36 & 1.14 \\
\hline SE & 0.35 & 0.29 & 0.25 & 0.35 & 0.21 & 0.21 & 0.29 & 0.17 & 0.23 \\
\hline $95 \% \mathrm{Cl}$ & $\begin{array}{c}-0.60 \text { to } \\
-0.89\end{array}$ & $\begin{array}{c}-0.55 \text { to } \\
-0.69\end{array}$ & $\begin{array}{c}-0.46 \text { to } \\
-0.60\end{array}$ & $\begin{array}{c}-0.54 \text { to } \\
-0.97\end{array}$ & $\begin{array}{c}-0.30 \text { to } \\
-0.59\end{array}$ & $\begin{array}{c}-0.25 \text { to } \\
-0.68\end{array}$ & $\begin{array}{c}-0.55 \text { to } \\
-0.69\end{array}$ & $\begin{array}{c}0.99 \text { to } \\
-1.72\end{array}$ & $\begin{array}{l}0.64 \text { to } \\
-1.64\end{array}$ \\
\hline$P$ & 0.34 & 0.4 & 0.39 & 0.28 & 0.25 & 0.17 & 0.4 & 0.0001 & 0.0001 \\
\hline Scan choice & Non & Non & Non & Non & Non & Non & Non & $3 D$ & $3 \mathrm{D}$ \\
\hline
\end{tabular}

The interleaved SFOV scan sequence reduced 2D or 3D measurement bias, and paired scans that were close in time resulted in insignificant tracer concentration redistribution and decay difference effects between acquisition modes. Because interleaved SFOV acquisitions did not require additional CT exposure beyond their initial WB CT used for attenuation correction, the PET/CT protocol minimized each patient's WB effective dose to ionizing radiation. If

TABLE 5.

Spearman Correlation Coefficient of SUVmax

\begin{tabular}{cccccc}
\hline & \multicolumn{5}{c}{$P$} \\
\cline { 2 - 6 } Scan & $\begin{array}{c}\text { Age at } \\
\text { scan }(\mathrm{y})\end{array}$ & $\begin{array}{c}\text { Weight } \\
(\mathrm{kg})\end{array}$ & $\begin{array}{c}\text { Height } \\
(\mathrm{m})\end{array}$ & $\begin{array}{c}\mathrm{BMl} \\
\left(\mathrm{kgm}^{-2}\right)\end{array}$ & $\begin{array}{c}\text { Blood glucose } \\
\left(\mathrm{mmol}^{-1}\right)\end{array}$ \\
\hline 2D & & & & & \\
$4 \mathrm{~min}$ & 0.82 & 0.97 & 0.18 & 0.12 & 0.45 \\
$3 \mathrm{~min}$ & 0.66 & 0.73 & 0.28 & 0.06 & 0.43 \\
2 min & 0.57 & 0.93 & 0.43 & 0.30 & 0.53 \\
3D & & & & & \\
4 min & 0.65 & 0.75 & 0.45 & 0.15 & 0.47 \\
3 min & 0.69 & 0.83 & 0.50 & 0.26 & 0.43 \\
2 min & 0.67 & 0.67 & 0.59 & 0.17 & 0.33
\end{tabular}

No significant correlations were observed between any 2D or 3D acquisition modes and scan duration with age, weight, height, BMI, or blood glucose. movement occurred between the WB scan and subsequent 2D and 3D SFOV acquisitions, then misregistration artifacts would likely have influenced both 2D and 3D scans equally. With our protocol, we could not account for movement between paired interleaved 2D and 3D scans. However, all SFOV images were investigated visually for gross misregistration, to minimize attenuation correction errors, and no errors were observed.

To minimize movement artifacts in head and neck cancer patients, routine 2D WB imaging (scan duration, $4 \mathrm{~min} / \mathrm{bed}$ position) was performed in the cranial to caudal direction, starting on average $65 \mathrm{~min}$ after the injection of the tracer. Head and neck lesions were seen during the first or second bed positions during scanning. The first 2D 4-min SFOV scan was obtained after the WB scans, on average $92 \mathrm{~min}$ after the injection of the tracer. The average delay between ending the head and neck lesion imaging part of the standard WB scan and starting the first optimized SFOV scan was $21 \mathrm{~min}$. This time delay had the potential to introduce some redistribution and decay bias in uptake between the routine clinical WB and research SFOV scans; however, no significant differences in mean SUVmax or SUVmean were observed $(P=0.28$ and $P=0.07$, respectively).

We validated the efficacy of data in this study by investigating tumor uptake bias and noted no correlation between SUVmax and age, weight, height, BMI, or blood glucose (Table 5). This result confirmed our expectation, because the 
average patient $\mathrm{BMI} \pm \mathrm{SD}(24.9 \pm 1.8)$ in our cohort did not include obese patients $(\mathrm{BMI}>30)$ in whom one might potentially expect more correlations. Incidentally, in obese subjects 2D imaging may be preferred because of increased photon scatter rejection using a collimator. To further authenticate our methodology and data, we compared like with like when contrasting 2D and 3D scans by noise-matching images between paired scans (8). A close match in noise between paired scans for 2, 3, and 4 min, respectively, was achieved (Fig. 3) before further data analysis.

No significant differences were subsequently seen between mean SUVmean in paired scans (Fig. 4). In addition, we observed no statistically significant differences between any individual mean 2D SUVmean of different scan times: 2D 4 -min vs. $2 \mathrm{D} 3$-min scans $(P=0.78), 2 \mathrm{D} 4-\mathrm{min}$ vs. $2 \mathrm{D}$ 2-min scans $(P=0.27)$, and 2D 3-min vs. 2D 2-min scans $(P=0.32)$. The same was true for individual mean $3 \mathrm{D}$ SUVmean and between all combinations of mean 2D SUVmean, compared with mean 3D SUVmean, with the same or different scan times.

For scan-time optimization in 2D mode quantitatively, we observed no significant difference between any of the mean SUVmax for different time intervals (Fig. 5): 2D 4-min vs. 2D 3-min scans $(P=0.41), 2 \mathrm{D} 4$-min vs. 2D 2-min scans $(P=0.93)$, or $2 \mathrm{D} 3$-min vs. $2 \mathrm{D} 2$-min scans $(P=0.50)$. Accordingly, the qualitative observer visual analysis scores reflected this result because no significant preference was found between 2D 4-, 3-, and 2-min scans. Furthermore, the difference in noise means of $1.91 \%$ between 2D 4-min scans and 2D 3-min scans was not significant $(P=0.07)$, whereas the corresponding 2D 4- and 2D 2-min difference of $6.24 \%$ was significant $(95 \% \mathrm{CI},-0.31$ to -0.01 ; paired $t$ test, $t=$ $-2.26, P=0.04)$. All these data support our pragmatic suggestion of a scan-time reduction per bed position in 2 dimensions from 4 to $3 \mathrm{~min}$ as routine for head and neck cancer; however, because our sample size and BMI were limited in this study, we would caution against routinely using $2 \mathrm{~min} / \mathrm{bed}$ position.

In the case of scan-time optimization in 3D mode quantitatively, we saw a significant difference in mean SUVmax between 3D 4-min scans and 3D 2-min scans only (95\% CI, -0.14 to -0.02 ; paired $t$ test, $t=-2.88, P=0.01$ ). Qualitative visual analysis scores for 3D mode showed no significant preferences for any scan time in comparison with another. Again, we can suggest 3D imaging routinely using $3 \mathrm{~min} /$ bed position acquisition in head and neck cancer but would urge caution against using $2 \mathrm{~min} / \mathrm{bed}$ position for the same reasons as declared above in the 2D case.

When comparing 2D with 3D scan modes quantitatively using paired SUVmax data of the same scan duration (Fig. 5), mean 2D SUVmax at 4-, 3-, and 2-min scan durations was greater in magnitude than equivalent mean 3D SUVmax. Nonetheless, only the 4-min difference between the mean 2D SUVmax and mean 3D SUVmax was significant $(P=0.01)$, suggesting a slight quantitative $2 \mathrm{D}$ scanning, compared with 3D scanning, advantage using SUVmax. However, compar- ison of visual analysis observer scores between 2D mode and $3 \mathrm{D}$ mode for the same scan time confirmed that 3 dimensions were significantly preferred over 2 dimensions at $4(P=$ $0.0001)$ and $3 \mathrm{~min}(P=0.0001)$ (Table 4), suggesting an overall qualitative advantage of $3 \mathrm{D}$ scans, compared with $2 \mathrm{D}$ scans, in head and neck cancer.

The results of the $2 \mathrm{D}, 3 \mathrm{D}$, and $2 \mathrm{D}$ versus $3 \mathrm{D}$ scans presented here illustrate the potential for slight advantages in $2 \mathrm{D}$ scanning quantitatively and in $3 \mathrm{D}$ scanning qualitatively, thus highlighting both the benefits of each mode and the lack of dominance of one mode over the other in head and neck imaging on this BGO system. The lack of significant difference in paired 2D and 3D SUVmean data further supports the equivalence of imaging modes and generally complements the SUVmax data.

However, SUVmax measurements are typically more reproducible than are SUVmean measurements, for which the entire lesion must be accurately delineated by an ROI for accurate values (20) and the influence of background in the ROI influences the result; therefore, we focus on SUVmax for quantitative analysis.

Our results are validated by comparable $2 \mathrm{D}$ and $3 \mathrm{D}$ outcomes in work performed by other groups. Congruent results of $2 \mathrm{D}$ and 3D quantitative image analysis have been presented in phantom studies on the same make and model of BGO scanner as the one used in this study, using widely accepted NEMA tests (21), and on more modern BGO systems (22). 2D and 3D mode equivalence is also true for qualitative analysis in WB clinical studies on an older BGO system (7); in addition, similar to our study, qualitative preference in 3D scans was expressed by physicians using current GE scanners (23) consisting of different crystals.

There were several limitations to this study. We performed our optimization process with the NEMA-recommended phantom using a hot sphere-to-background ratio of approximately $4: 1$, but there are other ratios of clinical significance (e.g., 2:1 ratio) relating to patient management.

The entire scan protocol was not simple to implement clinically; to reduce complication and possible errors in execution, we did not implement an inversion in the order of SFOV scans in half the patients. Consequently, we may have introduced some bias. However, for 3-min scans, which we recommend, the order of scans was reversed so that 2 dimensions followed 3 dimensions and the difference in mean SUVmax between these was not significant $(P=0.17)$, implying that scan order did not heavily bias results for our suggested scan duration of $3 \mathrm{~min}$.

To ensure convergence, our SFOV reconstruction parameters were at a higher number of iterations and subsets than standard WB imaging. However, there was no significant difference between head and neck lesion mean SUVmax or mean SUVmean between the standard WB and first SFOV reconstructed scans. Because we obtained the same results when performing the study without noise matching or optimization using standard SFOV reconstructions (i.e., 2 dimensions are quantitatively superior, and 3 dimensions 
are qualitatively preferred (24)), further validation is unwarranted.

We observed variations in the recorded data similar to those shown by others and believe these differences may be accounted for by a combination of factors. These include calibration errors between 2D scans and 3D scans, combined with differences in scatter-correction accuracy applied between acquisition modes. During image reconstruction, it may be possible that $3 \mathrm{D}$ scatter correction overcorrects in some cases, leading to reduced 3D uptake, whereas 2D SUVmax may be overemphasized in noisy backgrounds. Gaussian filter selection for 2D and 3D noise matching may have influenced spatial resolution and also played a role. However, this effect was variable, thereby reducing a potential systematic bias in resolution (25); no significant differences between standard WB and first SFOV lesion SUVmax or SUVmean were recorded.

In addition to these limitations, we advise some caution in applying the outcome of this clinical study to routine WB imaging and to other tumor sites. In this work, we investigated head and neck lesions in the center of the FOV and our protocol did not support the study of varied slice overlaps or other image-reconstruction parameters that influence 2D or 3D imaging. Moreover, none of the patients in this study was clinically obese and, hence, the potential bias for 2D scanning that might be the case in such conditions was unlikely in this study.

In support of our study, other studies that performed work without optimization, for example, those with a form of resolution matching (8) or more recently those without (13), acknowledged this drawback. Taking work performed without optimization into account, along with noise-matched images and the similarity of our results to those of others, this study is valid and overcomes the limitations presented.

3D imaging in this study generally favored lesion conspicuity, but all lesions were clearly seen in all patients regardless. Instead, we propose that 2D imaging and SUVmax quantitation are important parameters for optimization regarding scan time per bed position. Furthermore, no significant differences were seen between 2D 4-min SFOV and 2D 3-min SFOV acquisitions for mean SUVmax, mean SUVmean, or mean noise $(P=0.41, P=0.78$, and $P=$ 0.07 , respectively). Thus, we can recommend 2D 3-min scans as a pragmatic alternative to 2D 4-min acquisitions for reduced scan time per bed position in head and neck cancer.

\section{CONCLUSION}

In noise-matched paired scans, we have shown a slight quantitative advantage to $2 \mathrm{D}$ scanning, with higher mean SUVmax, and a qualitative advantage to 3D scanning, with preference for lesion conspicuity. However, all lesions were identified in all scans regardless of acquisition mode, and consequently there was no overwhelming advantage or disadvantage to using either acquisition mode. For quantitative studies in head and neck cancer, we recommend routine 2 D clinical imaging with a 3-min scan duration over an SFOV using this BGO-based PET/CT scanner with our imaging protocol and reconstruction defaults.

\section{ACKNOWLEDGMENTS}

We thank all staff for performing complex scans; Professor Brian Hutton, from University College Hospital, for providing image reconstruction advice; and, posthumously, Helmut Bammer, from GE, for providing his scanner technical input and support.

\section{REFERENCES}

1. Knesaurek K. New developments in PET instrumentation: quo vadis PET? J Nucl Med. 2001;42:1831-1832.

2. Gundlich B, Musmann P, Weber S, Nix O, Semmler W. From 2D PET to 3D PET: issues of data representation and image reconstruction. $Z$ Med Phys. 2006;16:31-46.

3. Lartizien C, Kinahan PE, Swensson R, et al. Evaluating image reconstruction methods for tumor detection in 3-dimensional whole-body PET oncology imaging. J Nucl Med. 2003;44:276-290.

4. Lartizien C, Comtat C, Kinahan PE, Ferreira N, Bendriem B, Trébossen R. Optimization of injected dose based on noise equivalent count rates for 2- and 3-dimensional whole-body PET. J Nucl Med. 2002;43:1268-1278.

5. Raylman RR, Kison PV, Wahl RL. Capabilities of two- and three- dimensional FDG-PET for detecting small lesions and lymph nodes in the upper torso: a dynamic phantom study. Eur J Nucl Med. 1999;26:39-45.

6. Lartizien C, Kinahan PE, Comtat CA. Lesion detection observer study comparing 2-dimensional versus fully 3-dimensional whole-body PET imaging protocols. J Nucl Med. 2004;45:714-723.

7. Visvikis D, Griffiths D, Costa DC, Bomanji J, Ell PJ. Clinical evaluation of 2D versus 3D whole-body PET image quality using a dedicated BGO PET scanner. Eur J Nucl Med Mol Imaging. 2005;32:1050-1056.

8. Lodge MA, Badawi RD, Gilbert R, Dibos PE, Line BR. Comparison of 2-dimensional and 3-dimensional acquisition for ${ }^{18} \mathrm{~F}$-FDG PET oncology studies performed on an LSO-based scanner. J Nucl Med. 2006;47:23-31.

9. Dhawan V, Kazumata K, Robeson W, et al. Quantitative brain PET: comparison of $2 \mathrm{D}$ and $3 \mathrm{D}$ acquisitions on the GE Advance scanner. Clin Positron Image. 1998;1:135-144.

10. Knesaurek K, Machac J, Krynyckyi RB, Almeida OD. Comparison of 2-dimensional and 3-dimensional ${ }^{82} \mathrm{Rb}$ myocardial perfusion PET imaging. $J$ Nucl Med. 2003;44:1350-1356.

11. Votaw JR, White M. Comparison of 2-dimensional and 3-dimensional cardiac ${ }^{82}$ Rb PET studies. J Nucl Med. 2001;42:701-706.

12. Lubberink M, Boellaard R, van der Weerdt AP, Visser FC, Lammertsma AA. Quantitative comparison of analytic and iterative reconstruction methods in 2- and 3-dimensional dynamic cardiac ${ }^{18}$ F-FDG PET. J Nucl Med. 2004;45:2008-2015.

13. Komar G, Teras M, Seppanen M, et al. Comparison of 2D and 3D performance for FDG PET with different acquisition times in oncological patients. Nucl Med Commun. 2009;30:16-24.

14. Sanghera B, Wong WL, Lodge MA, et al. Potential novel application of dual time point SUV measurements as a predictor of survival in head and neck cancer. Nucl Med Commun. 2005;26:861-867.

15. Dische S, Saunders M, Barrett A, Harvey A, Gibson D, Parmar M. A randomised multicentre trial of CHART versus conventional radiotherapy in head and neck cancer. Radiother Oncol. 1997;44:123-136.

16. Hudson HM, Larkin RS. Accelerated image reconstruction using ordered subsets of projection data. IEEE Trans Med Imag. 1994;13:601-609.

17. Bergstom M, Eriksson L, Bohm C, Blomqvist G, Litton J. Correction for scattered radiation in a ring detector positron camera by integral transformation of the projections. Comput Assist Tomogr. 1983;7:42-50.

18. Stearns CW, Fessler JA. 3D PET reconstruction with FORE and WLS-OS-EM. IEEE Nucl Sci Symp Conf Rec. 2002;2:912-915

19. Ollinger JM. Model-based scatter correction for fully 3D PET. Phys Med Biol. 1996;41:153-176.

20. Keyes JW Jr. SUV: standard uptake or silly useless value? J Nucl Med. 1995;36:1836-1839.

21. Mawlawi O, Podoloff D, Kohlmyer S, et al. Performance characteristics of a newly developed PET/CT scanner using NEMA standards in 2D and 3D modes. J Nucl Med. 2004;45:1734-1742. 
22. Teräs M, Tolvanen T, Johansson J, William J, Knuuti J. Performance of the new generation of whole-body PET/CT scanners: Discovery STE and Discovery VCT. Eur J Nucl Med Mol Imaging. 2007;34:1683-1692.

23. Mawlawi O, Truong M, Marom E, et al. Clinical evaluation of $2 \mathrm{D}$ versus $3 \mathrm{D}$ whole body FDG imaging performed on two PET scanners with different detectors [abstract]. J Nucl Med. 2007;489(suppl 2):442P.
24. Sanghera B, Lowe J, Lowe G, et al. Clinical study of $2 \mathrm{D}$ and $3 \mathrm{D}$ scan time reduction in head/neck cancer with BGO based PET/CT using statistical image analysis. IEEE Nucl Sci Symp Conf Rec. 2006;5:2658-2661.

25. Schoder H, Erdi YE, Chao K, et al. Clinical implications of different image reconstruction parameters for interpretation of whole-body PET studies in cancer patients. J Nucl Med. 2004;45:559-566.

\section{Erratum}

In the article "An Automated Method for Delineating a Reference Region Using Masked Volumewise PrincipalComponent Analysis in ${ }^{11}$ C-PIB PET," by Razifar et al. ( $\mathrm{N}$ Nucl Med Technol. 2009;37:38-44), the figure panel letters in the legends of Figures 3 and 4 were incorrect. The MVW-PC1 images were, in fact, shown in panels A, D, and G; the MVW-PC2 images in panels B, E, and H; and the MVW-PC3 images in panels C, F, and I. The authors regret the error. 